\title{
Yournal of Concer
}

$2011 ; 2: 132-135$

Case Report

\section{Adult Wilms Tumor with Intracaval and Intracardiac Extension: Report of a Case and Review of Literature}

\author{
Aitao Guo, Lixin Wei, Xin Song, Aijun Liu ${ }^{凶}$ \\ Department of Pathology, The General Hospital of PLA, Beijing 100853, China
}

Corresponding author: Dr. Aijun Liu, Department of Pathology, the General Hospital of PLA, Beijing 100853, China. liuaj@301hospital.com.cn. Telephone: +86-10-66936253

(C) Ivyspring International Publisher. This is an open-access article distributed under the terms of the Creative Commons License (http://creativecommons.org/ licenses/by-nc-nd/3.0/). Reproduction is permitted for personal, noncommercial use, provided that the article is in whole, unmodified, and properly cited

Received: 2011.01.26; Accepted: 2011.03.01; Published: 2011.03.02

\begin{abstract}
Wilms tumor (WT) occurs infrequently in adults. Even rarer is adult WT with extension by direct intravascular spread into the right side of the heart. The present report describes a partially differentiated WT with intracaval and intracardiac extension in a 54-year-old man. The morphologic and immunohistochemical findings confirmed the diagnosis.
\end{abstract}

Key words: Wilms tumor, adult, intracaval extension, intracardiac extension

\section{Introduction}

Wilms tumor is the most common primary malignant renal tumor in children, accounting for $6 \%$ of all pediatric malignant tumors [1], however, it is very rare in adults, with an incidence of about 0.2 per million per year in the USA and Europe [2]. Only approximately 300 well-documented cases of adult WT have been reported in the literature to date [3].

In children, invasion of the renal vein is present in about $12 \%$ of cases. Up to $4 \%$ of patients with WT have tumor thrombus involving the vena cava, and the incidence of cardiac involvement is about $0.8 \%$ to $1 \%$ [4, 5]. In adults, tumor extension into the vena cava and the right side of the heart is extremely rare. To our knowledge, only one case of an adult with caval involvement has been reported in the English-language literature [6].

In this report, a case of favorable histology WT with invasion of the inferior vena cava and extension to the right atrium is discussed with a review of the literature.

\section{Case Report}

A 54-year-old man presented to a local hospital with right flank pain that was believed to be caused by cholecystitis. And abdominal ultrasound revealed a hypo-echoic area measuring $2.5 \mathrm{~cm} \times 2.3 \mathrm{~cm}$ in the right renal collecting system. The patient denied back pain, gross hematuria, urinary frequency, urinary urgency, and urodynia.

Computerized tomography (CT) scan and magnetic resonance imaging (MRI) revealed a mass in the right kidney with extension into the right renal vein and inferior vena cava, and further extension into the right atrium (Fig. 1A). Retrograde urography confirmed that the right renal pelvis was compressed and distorted, but was unobstructed. The right ureter also was unobstructed. Digital subtraction angiography revealed tumor thrombus in the inferior vena cava extending from the level of the right renal vein to the right atrium (Figs 1B). Laboratory investigations revealed no abnormalities. Renal function tests were normal. Urine analysis revealed no microscopic hematuria.

The patient was transferred to our hospital for further evaluation and treatment. Based on the clinical findings and investigations, a preliminary diagnosis of renal carcinoma of the right kidney was made. The patient underwent a right radical nephrectomy in a 
thoraco-abdominal procedure. At the same time, the tumor thrombus was removed from the inferior vena cava and the right atrium.

The resected right kidney measured $14 \mathrm{~cm} \times 8 \mathrm{~cm} \times 6 \mathrm{~cm}$. Near the renal pelvis was a tumor measuring $7 \mathrm{~cm} \times 5 \mathrm{~cm} \times 4.5 \mathrm{~cm}$ with extension into the renal vein. On cut section, the mass had a solid and multicystic appearance with focal invasion of the renal parenchyma. The cysts measured from $0.5 \mathrm{~cm}$ to $1.5 \mathrm{~cm}$ in diameter, and were filled with clear colorless fluid. The solid area was gray and uniform, without necrosis or hemorrhage. The tumor distorted but did not infiltrate into the renal pelvis. The paraortic lymph nodes and the right adrenal gland showed no evidence of tumor. Macroscopically, the tumor thrombus measured $25 \mathrm{~cm} \times 1.5 \mathrm{~cm} \times 1.5 \mathrm{~cm}$, and was gray with a myxoid cut surface.

Histologically, the tumor exhibited a triphasic pattern of blastema, epithelium, and stroma with myxoid background in the solid area and in the septa of the cystic area (Figs 2). The lining cells of the cysts ranged from flattened to columnar, occasionally of "hobnail" type, and showed positive staining for cytokeratin (Fig 3A) and EMA. Interestingly, the majority of the cysts had distinct walls composed of spindle or oval cells that expressed muscle-derived markers, such as smooth muscle actin (1A4) and muscle actin (HHF35) (Fig 3B). There were no anaplastic components. Focal renal parenchymal invasion could be seen. The histologic features of the thrombus were similar to those of the renal tumor. Thus, the final diagnosis was favorable histology WT (partially differentiated type), with renal vein invasion and extension to the inferior vena cava and right atrium.

The patient received nine courses of adjuvant chemotherapy postoperatively, including two courses with Dactinomycin, Adriamycin, and Vincristine, two courses with Paclitaxel and Carboplatin, four courses with Paclitaxel, Ifosfamide, and Mesna, and one course with Vinorelbine and Dactinomycin. Multiple metastases to the liver, lung, and chest wall were occurred within 12 months after surgery, and the patient was dead from the disease 14 months later.

\section{Discussion}

WT is the most common primary malignant renal neoplasm of childhood, but it is rarely found in adults. Less than $1 \%$ of all WTs are found in adults. The preoperative diagnosis of adult WT is extremely difficult because there are no specific radiographic findings that can distinguish it from the more common adult malignant renal neoplasms [7]. Histopathologically, there is no difference between adult and childhood WTs. Both characteristically have tri- phasic histology, with components of blastemal, epithelial, and stromal structures, as were noted in our case. Pathological diagnosis of adult WT is also more difficult than in pediatric one, because there are many other adult renal tumors that should be considered in the differential diagnosis. Insufficient sampling of the tumor for histologic evaluation, and the absence of a systemic work-up can lead to misidentification of the tumor. Kilton et al [8] have applied six rigid diagnostic criteria for adult WT, which remain in use: 1) primary renal neoplasm, 2) primary blastematous spindle- or round-cell component, 3) formation of abortive or embryonal, tubular epithelial, or glomeruloid structure, 4) no areas of tumor diagnostic of renal cell carcinoma, 5) pictorial confirmation of histology and 6) age $>15$ years. According to these strict diagnostic criteria, only about 300 cases of adult WT have been reported in the English literature to date [4]. In the current case, the classic triphasic pattern was clear, with no areas suggestive of renal cell carcinoma. Thus, the final diagnosis was adult WT.

$4 \%$ children WT have caval involvement, and $0.8-1 \%$ of these patients also have atrial extension of the tumor $[4,5]$, However, the incidence of caval involvement in adults is unknown, and only one adult case with right atrial extension has been reported in English-language literature [6]. Echocardiography and color flow doppler ultrasound are helpful in detecting inferior vena caval and right atrial tumor if the WT has spread through the inferior vena cava into the right atrium [9]. The present case was diagnosed as inferior vena cava and right atrium involvement by the CT and MRI examination, although tumor type could not define before surgery.

Cases with intravascular involvement may represent a daunting challenge for the surgeon. In Mart's [10] experience, the surgical strategy for these cases depends on the length of the thrombus and whether or not the thrombus has infiltrated the wall of the vena cava. If the thrombus can be easily removed, complete resection is the treatment of choice, however, in cases of atrial thrombus, and more particularly if the thrombus involves the intima, these authors suggest that the thrombus not be touched, and the tumor should be treated by preoperative and postoperative chemotherapy.

The prognosis for adult WT is significantly worse than that for children [2]. This observation is only partially explained by the fact that more adults seem to have advanced stage at the time of diagnosis. Even when comparing tumors with same stage, the prognosis still appears to be worse in adults than in children. Kalapurakal et al. [11] have suggested that venous invasion correlates with the children's prog- 
nosis. In cases staged as N0M0V2a (in which thrombus is free in the vena cava, without infiltration of the wall), nephrectomy and unblocking of the abdominal vena cava may achieve remarkably long survival, however tumors of substage $\mathrm{V} 2 \mathrm{~b}$ (in which the thrombus infiltrates the wall of the vena cava) should be treated as tumors with visceral metastases.

Due to the rarity of WT in adults, no firm treatment guidelines have been established to date. Adult WT is treated according to recent pediatric protocols. In earlier reports it was agreed that all stages of adult
WT warrant aggressive multimodal therapy [surgery, chemotherapy, and radiotherapy] because of its poor prognosis. The National Wilms' Tumor Study Group (NWTSG) in the United State recommended that all adult patient with favorable histology should be treated with stage-appropriate combined therapy, as is done for WT in children [12]. The presented patient died from multiple metastases within 14 months although treated with adjuvant chemotherapy, implying that adult WT has poor prognosis and aggressive multimodal therapy should be scheduled.
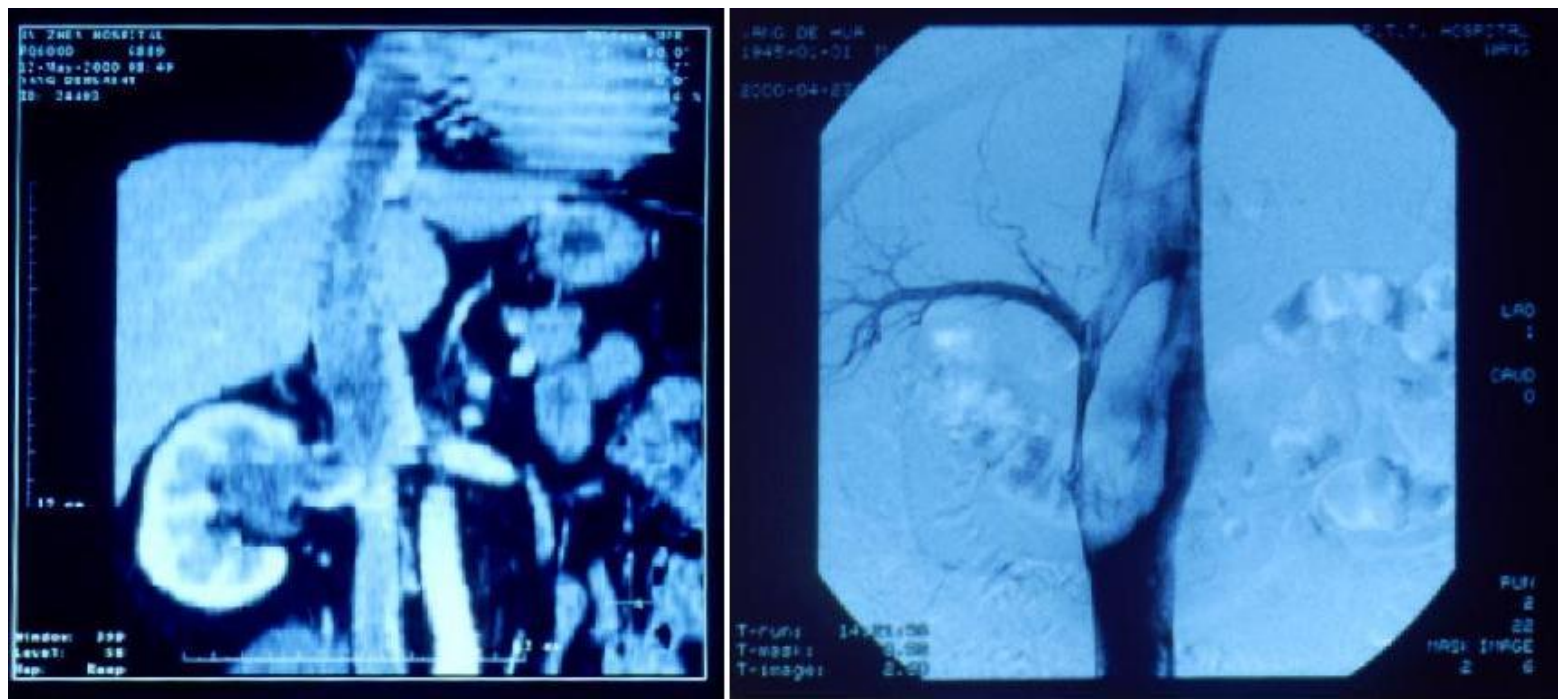

Figure I. Multi-plana Reconstruction of CT scan before operation showing a mass in the right renal area and propagation through the inferior vena cava into the right atrium IA (left). Digital subtraction angiography performed before surgery showing huge tumoral thrombus in the inferior vena cava extension upward from over the right renal vein level IB (right).
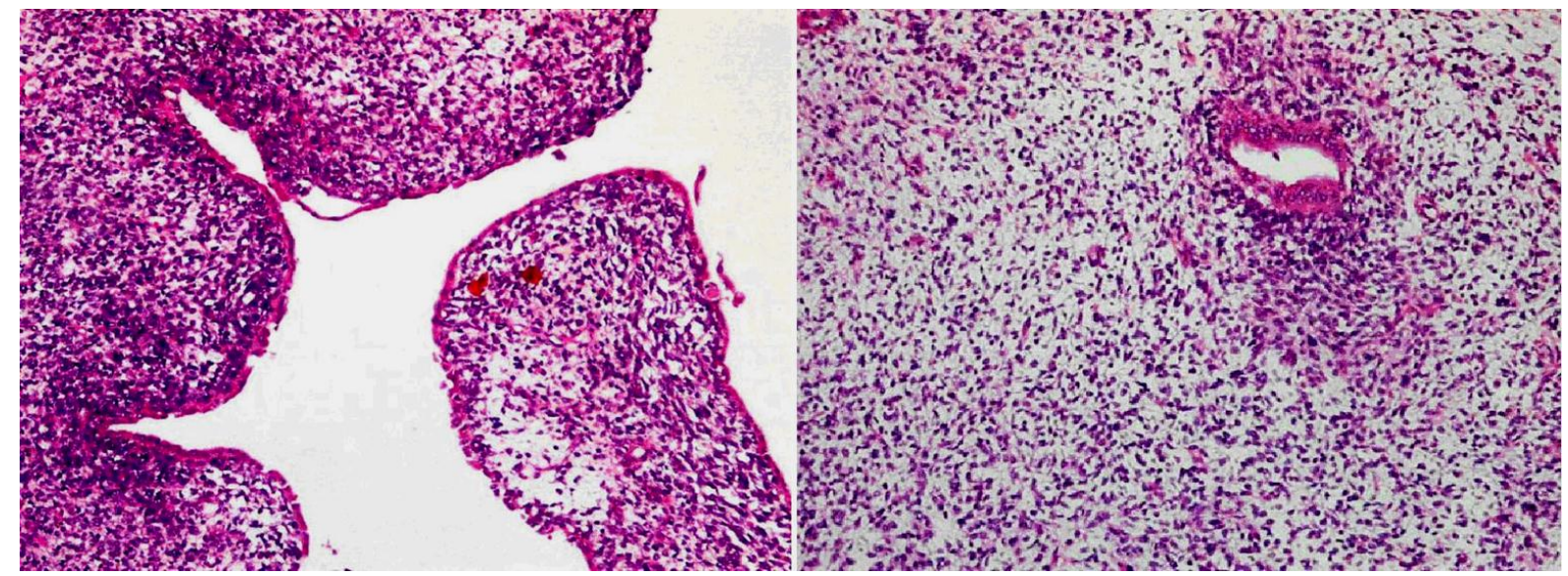

Figure 2. Histological examination of the adult WT. 2A (left) representative of the cystic area. The lining cells of the cysts, ranged from flattened to columnar, were occasionally of "hobnail" type, and the cysts had distinct walls composed of spindle or oval cells. 2B (right) showing a mixture of blastemal and epithelial components with myxoid background in the solid area. [x100] 


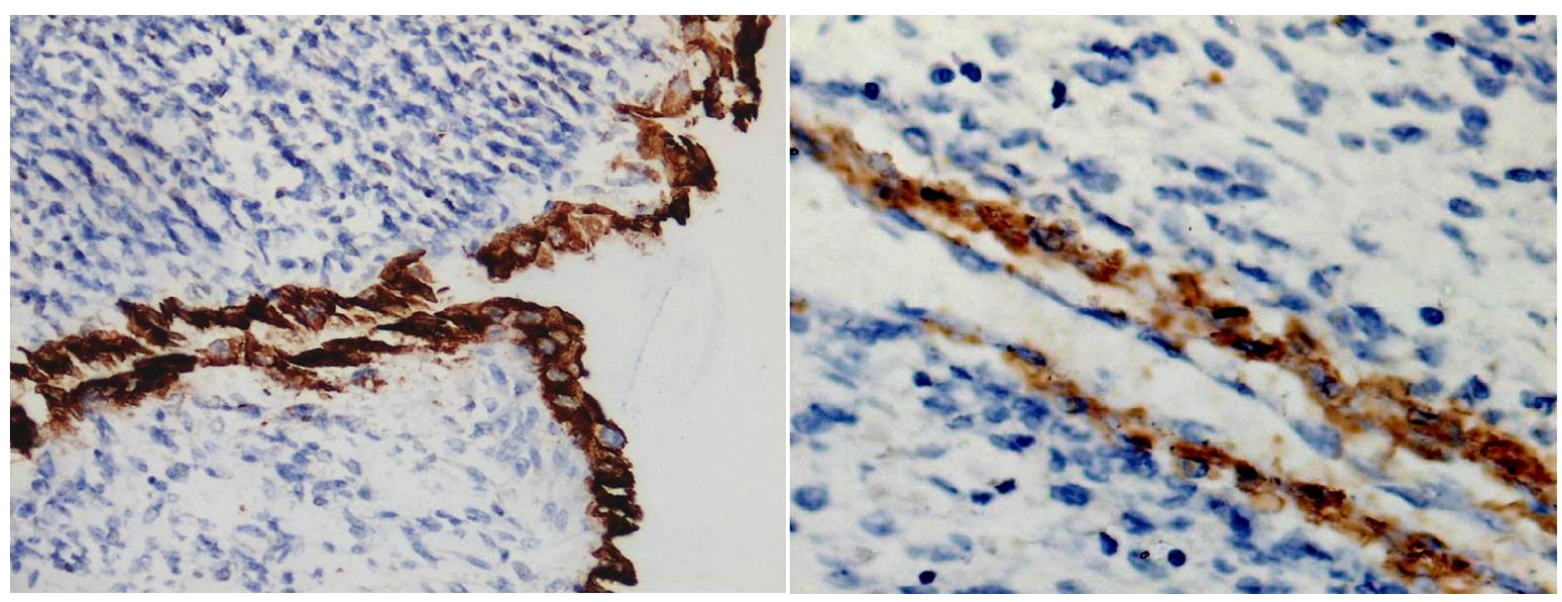

Figure 3. immunohistochemistry staining of the adult WT. 3A (left) The lining cells of the cysts showed positive staining for cytokeratin and 3B (right) the spindle or oval cells of the cysts walls expressed muscle actin [ $\times 200]$.

\section{Conflict of Interest}

The authors have declared that no conflict of interest exists.

\section{References}

1. Zugor V, Schott GE, Lausen B, et al. Clinical and surgical experience with Wilms tumor. Long-term results of a single institution. Anticancer Res. 2010; 30: 1735-1739

2. Mitry E, Ciccolallo L, Coleman MP, et al. Incidence of and survival from WILMS TUMOR in adults in Europe: Data from the EUROCARE study. Eur J Cancer. 2006;42:2363-2368

3. Terenziani M, Spreafico F, Collini P, et al. Adult Wilms Tumor: A monoinstitutional experience and a review of the literature. Cancer. 2004; 101:289-293

4. Namboodiri N, Krishnamoorthy KM, Tharakan JA. Intra-atrial extension of Wilms' Tumor. J Am Soc Echocardiogr. 2008; 21:91e3-4

5. Szavay P, Luithle T, Semler O, et al. Surgery of cavoatrial tumor thrombus in nephroblastoma: a report of the SIOP/GPOH Study. Pediatr Blood Cancer. 2004; 43: 40-45
6. Fonseca P, Almassi GH, Begun F. Resection of an adult intracardiac Wilms Tumor using Hypothermic circulation arrest. Ann Thorac Surg. 1995; 60: 1818-1820

7. Kaur N, Gupta A, Attam A, et al. Adult Wilms Tumor: management considerations. Int Urol Nephrol. 2005; 37:17-20

8. Kilton L, Matthews MJ, Cohen MH. Adult Wilms Tumor: a report of prolonged survival and review of literature. J Urol.1980; 124: 1-5

9. Solwa Y, Sanyika C, Hadley GP,. et al. Colour Doppler ultrasound assessment of the inferior vena cava in patients with Wilms Tumor' tumor. Clin Radiol. 1999; 54: 811-814

10. Martínez-Ibáñez V, Sánchez de Toledo J. et al. Wilms Tumor' tumor with intracaval involvement. Med Pediatr Oncol. 1996; 26: $268-271$

11. Kalapurakal JA, Nan B, Norkool P, et al. Treatment outcomes in adults with favorable histologic type Wilms Tumor-an update from the National Wilms Tumor Study Group. Int J Radiat Oncol Bio Phys. 2004; 60: 1379-1384

12. Reinhard $H$, Aliani $S$, Ruebe $C$, et al. Wilms Tumor in adults: results of the Society of Pediatric Oncology [SIOP] 93-01 / Society for Pediatric Oncology and Hematology [GPOH] Study. J Clin Oncol 2004; 22: 4500-4506 